\title{
High order mode power loss evaluation in future circular electron-positron collider cavities
}

\author{
Ivan Karpov, Rama Calaga, and Elena Shaposhnikova \\ CERN, CH 1211 Geneva 23, Switzerland
}

(Received 24 April 2018; published 26 July 2018)

\begin{abstract}
The high order mode (HOM) power losses induced by a particle beam in accelerator components should be taken into account during their design and the choice of HOM couplers. In this work the power losses due to HOMs in the proposed $400.79 \mathrm{MHz}$ radio frequency (rf) cavity designs were evaluated for various energy options $(\mathrm{Z}, \mathrm{W}, \mathrm{H}$, and $\overline{\mathrm{t}})$ of the future circular electron-positron collider (FCC-ee). The constraints for the rf cavity design are dictated by the $\mathrm{Z}$ machine with the lowest beam energy, but the highest beam current. Still certain beam filling patterns (bunch and train spacings) are not feasible and should be avoided in beam operation. For other energy options the power loss are sufficiently low and can be extracted by conventional HOM couplers.
\end{abstract}

DOI: 10.1103/PhysRevAccelBeams.21.071001

\section{INTRODUCTION}

The rf power and heat load deposited by a beam passing through the accelerator structures can cause significant limitations for the machine performance. To determine parameters for high order mode (HOM) absorbers accurate estimations of the power loss are required, which depend both on the beam parameters and the geometric design of $\mathrm{rf}$ cavities. The power loss can be calculated as $[1,2]$

$$
P=J_{A}^{2} \sum_{k=-\infty}^{+\infty} \operatorname{Re}\left[Z_{\|}\left(k \omega_{0}\right)\right]\left|\hat{J}_{k}\right|^{2},
$$

where $J_{A}$ is the average beam current, $Z_{\|}$is the longitudinal impedance, $\hat{J}_{k}$ is the normalized Fourier harmonic of the beam current at the $k$ th revolution harmonic, $\omega_{0}=2 \pi f_{0}$, and $f_{0}$ is the revolution frequency.

The evaluation of the power losses in superconducting rf cavities for FCC-ee is the subject of the present work. For calculations we used the modified design of the large hadron collider (LHC) rf cavities and the latest parameter set of different FCC-ee machines summarized in Table I [3]. We applied a systematic approach to power loss calculation taking into account different filling schemes used in machine operation. This allows us to derive recommendations for the both cavity design and machine operation.

Published by the American Physical Society under the terms of the Creative Commons Attribution 4.0 International license. Further distribution of this work must maintain attribution to the author(s) and the published article's title, journal citation, and DOI.
The analysis of Fourier harmonics for different filling patterns of the beam with a Gaussian bunch line density is presented in Sec. II. The longitudinal impedance and the loss factor of cavities obtained from electromagnetic (EM) simulations are described in Sec. III. In Sec. IV we present the results of power loss calculations for different cavity options and all FCC-ee machines. In the case when beam spectral line coincides with a cavity resonance one can expect strong power losses. We propose the way to avoid such situations. The work is concluded in Sec. V.

\section{BEAM SPECTRUM CALCULATION}

Let us consider a beam containing $M$ bunches which can be grouped in $n_{\text {tr }}$ equal trains with a distance between heads of the trains $t_{\mathrm{tt}}$. This distance should be a multiple of bunch spacing $t_{\mathrm{bb}}$. Each train contains a number of filled buckets $M_{\mathrm{b}} \leq t_{\mathrm{tt}} / t_{\mathrm{bb}}$. The beam has a regular filling which also contains an abort gap of length $t_{\text {gap }}$. Arbitrary filling schemes would require additional tedious analysis with many degrees of freedom and therefore are not considered in the present work.

The time structure of the beam current $J(t)$ can be written, similarly to Ref. [4],

$$
J(t)=\sum_{n=0}^{n_{\mathrm{tr}}-1} \sum_{m=0}^{M_{\mathrm{b}}-1} j\left(t-n t_{\mathrm{tt}}-m t_{\mathrm{bb}}\right)=J_{A} \sum_{k=-\infty}^{\infty} \hat{J}_{k} e^{i 2 \pi k f_{0} t},
$$

where $j(t)$ is the single bunch current and $J_{A}=$ $n_{\mathrm{tr}} M_{\mathrm{b}} N_{\mathrm{p}} e f_{0}$ is the average beam current. The normalized Fourier harmonics of the beam current can be calculated as 
TABLE I. FCC-ee baseline parameters [3] used for power loss calculations in this work. The bunch length is given for the cases of colliding beams including beamstrahlung (BS) effect and noncolliding beams (no BS).

\begin{tabular}{|c|c|c|c|c|c|}
\hline Parameter & Units & $\mathrm{Z}$ & $\mathrm{W}$ & $\mathrm{H}$ & $\mathrm{t} \overline{\mathrm{t}}$ \\
\hline Circumference, $C$ & $\mathrm{~km}$ & \multicolumn{4}{|c|}{97.75} \\
\hline Harmonic number, $h$ & & \multicolumn{4}{|c|}{130680} \\
\hline rf frequency, $f_{\text {rf }}$ & $\mathrm{MHz}$ & \multicolumn{4}{|c|}{400.79} \\
\hline Beam energy, $E$ & $\mathrm{GeV}$ & 45.6 & 80 & 120 & 182.5 \\
\hline Beam current, $J_{A}$ & $\mathrm{~mA}$ & 1390 & 147 & 29 & 5.4 \\
\hline $\begin{array}{l}\text { Number of bunches } \\
\text { per beam, } M\end{array}$ & & 16640 & 2000 & 328 & 48 \\
\hline Bunch population, $N_{\mathrm{p}}$ & $10^{11}$ & 1.7 & 1.5 & 1.8 & 2.3 \\
\hline rms bunch length (BS), $\sigma$ & ps & 40.4 & 20.0 & 17.7 & 8.5 \\
\hline $\begin{array}{l}\text { rms bunch length } \\
\text { (no BS), } \sigma\end{array}$ & ps & 11.7 & 10.0 & 10.5 & 6.6 \\
\hline $\begin{array}{l}\text { Momentum compaction } \\
\text { factor, } \alpha_{p}\end{array}$ & $10^{-6}$ & 14.79 & 14.79 & 7.31 & 7.31 \\
\hline Synchrotron tune, $Q_{s}$ & & 0.025 & 0.051 & 0.036 & 0.087 \\
\hline $\begin{array}{l}\text { Longitudinal damping } \\
\text { time, } \tau_{\mathrm{SR}}\end{array}$ & $\mathrm{ms}$ & 415.1 & 76.9 & 22.9 & 6.7 \\
\hline Total rf voltage, $V_{\text {tot }}$ & GV & 0.1 & 0.75 & 2 & 10.9 \\
\hline
\end{tabular}

$$
\hat{J}_{k}=\frac{1}{J_{A} T_{0}} \int_{-T_{0} / 2}^{T_{0} / 2} \sum_{n=0}^{n_{\mathrm{tr}}-1} \sum_{m=0}^{M_{\mathrm{b}}-1} j\left(t-n t_{\mathrm{tt}}-m t_{\mathrm{bb}}\right) e^{-i 2 \pi k f_{0} t} d t,
$$

where $T_{0}=1 / f_{0}$ is the revolution period. Using the normalized Fourier harmonics of the single bunch $\hat{j}_{k}$, this equation can be rewritten in a form

$$
\hat{J}_{k}=\hat{j}_{k} \sum_{n=0}^{n_{\mathrm{tr}}-1} e^{-i 2 \pi k f_{0} n t_{\mathrm{tt}}} \sum_{m=0}^{M_{\mathrm{b}}-1} e^{-i 2 \pi k f_{0} m t_{\mathrm{bb}}} .
$$

After summations in Eq. (4), the normalized Fourier harmonics of the beam current are

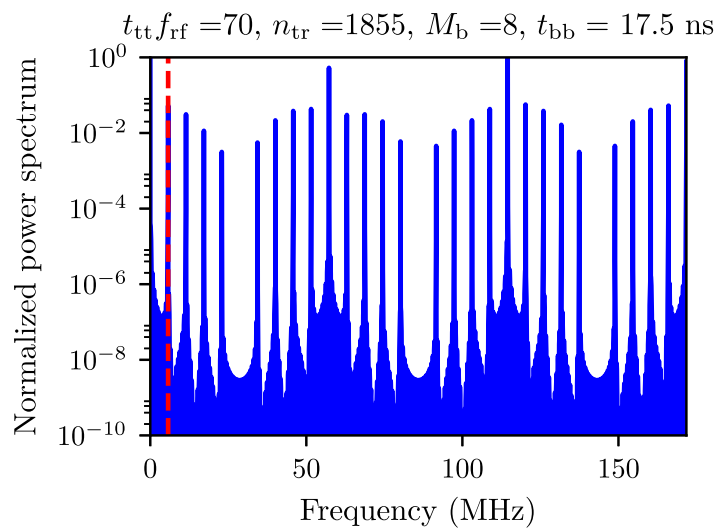

$$
\begin{aligned}
\hat{J}_{k}= & \hat{j}_{k} \times \frac{\sin \left(n_{\mathrm{tr}} \pi k f_{0} t_{\mathrm{tt}}\right)}{n_{\mathrm{tr}} \sin \left(\pi k f_{0} t_{\mathrm{tt}}\right)} e^{-i\left(n_{\mathrm{tr}}-1\right) \pi k f_{0} t_{\mathrm{tt}}} \\
& \times \frac{\sin \left(M_{\mathrm{b}} \pi k f_{0} t_{\mathrm{bb}}\right)}{M_{\mathrm{b}} \sin \left(\pi k f_{0} t_{\mathrm{bb}}\right)} e^{-i\left(M_{\mathrm{b}}-1\right) \pi k f_{0} t_{\mathrm{bb}}}
\end{aligned}
$$

For a Gaussian bunch with the rms bunch length $\sigma$,

$$
j(t)=\frac{e N_{\mathrm{p}}}{\sqrt{2 \pi} \sigma} \exp \left\{-\frac{t^{2}}{2 \sigma^{2}}\right\}
$$

and

$$
\hat{j}_{k}=\frac{1}{e N_{\mathrm{p}}} \int_{-T_{0} / 2}^{T_{0} / 2} j(t) e^{-i 2 \pi k f_{0} t} d t \approx e^{-2\left(\pi k f_{0} \sigma\right)^{2}} .
$$

For a fixed minimum gap length and the maximum total number of bunches, the number of trains and the number of bunches per train are defined by train spacing as

$$
n_{\mathrm{tr}}=\left\lfloor\frac{T_{0}-t_{\mathrm{gap}}}{t_{\mathrm{tt}}}\right\rfloor \quad \text { and } \quad M_{b}=\left\lfloor\frac{M}{n_{\mathrm{tr}}}\right\rfloor,
$$

correspondingly, where $\lfloor x\rfloor$ denotes a rounded down value of $x$.

There are two sets of lines with the largest amplitude in the beam spectrum: (i) at the multiple of frequencies $1 / t_{\mathrm{tt}}$ [the second part of Eq. (5)] given by the train spacing, (ii) at the multiple of frequencies $1 / t_{\mathrm{bb}}$ [the third part of Eq. (5)] given by the bunch spacing.

The $\mathrm{Z}$ machine is the most challenging due to high beam current. Depending on the filling scheme one can define two different regimes. The spectrum of the beam containing the large number of trains $\left(n_{\mathrm{tr}} \gg M_{\mathrm{b}}\right)$ is dominated by lines with the distance $1 / t_{\mathrm{tt}}$ (the left-hand side plot in Fig. 1). For the case of long trains $\left(n_{\mathrm{tr}} \leq M_{\mathrm{b}}\right)$, the spectrum mainly contains lines at harmonics of $1 / t_{\mathrm{bb}}$ corresponding to the bunch spacing. The maximum amplitude reduction of

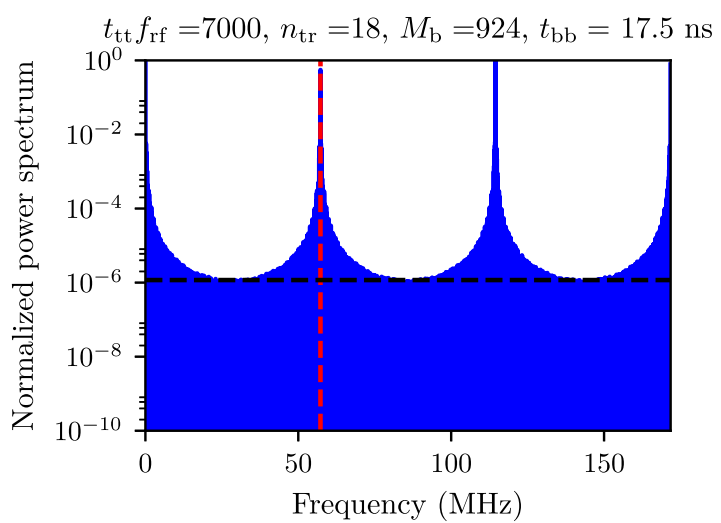

FIG. 1. Examples of the normalized beam spectra (parameters of the $\mathrm{Z}$ machine) for two regimes. The left-hand side plot is for the case of the large number of short trains. The red line is at $1 / t_{\mathrm{tt}}$. The right-hand side plot is the case of long trains. The red line is at $1 / t_{\mathrm{bb}}$, and the horizontal black line is $1 / M_{\mathrm{b}}^{2}$. 

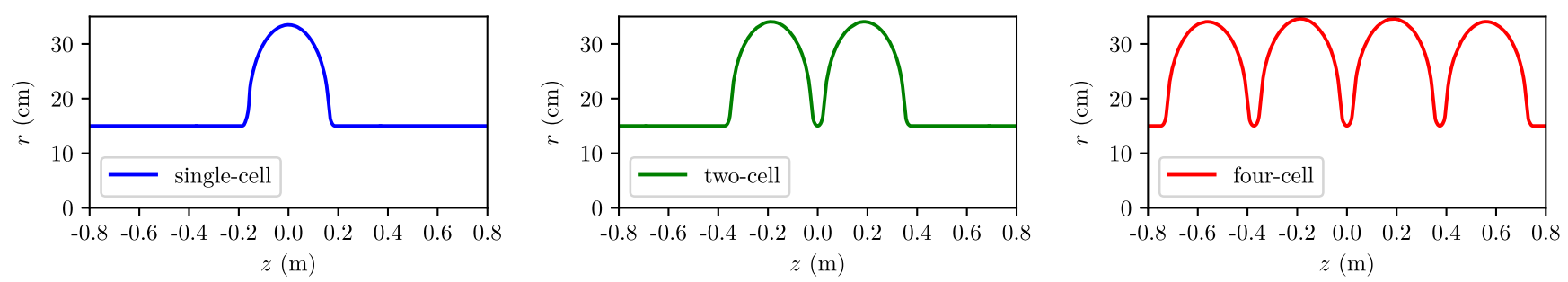

FIG. 2. LHC-like cavity designs with different number of cells (single-cell, two-cell, and four-cell cavities).

spectral lines between them is $1 / M_{\mathrm{b}}$ (the right-hand side plot in Fig. 1).

\section{IMPEDANCE CALCULATION}

The $400 \mathrm{MHz}$ LHC-like cavities [5] with different number of cells (single-cell, two-cell, and four-cell cavities) were considered in the present study of the FCC-ee rings (Fig. 2). Four cavities of one of these types with two tapers at the ends are placed in a single cryomodule (Fig. 3). The taper-out corresponds to the transition from the smaller radius $b$ to a larger radius $d$ (in the direction of beam motion) and the taper-in vice versa. The ABCI code [6] was

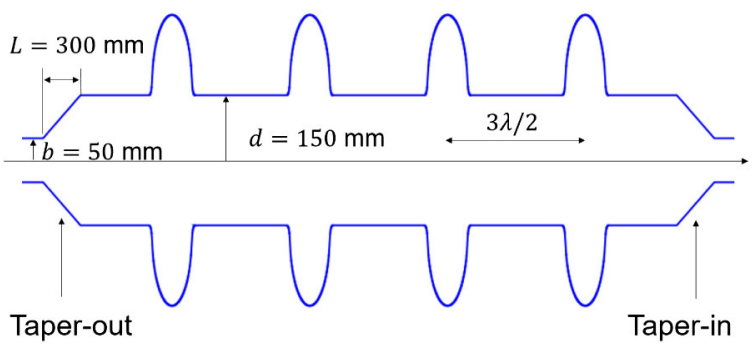

FIG. 3. Example of the LHC-like cryomodule containing four single-cell cavities and tapers [7].

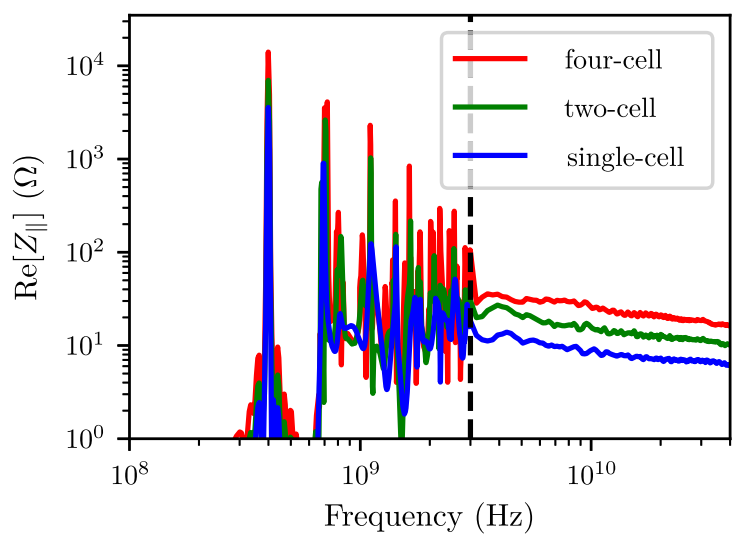

FIG. 4. The real part of longitudinal impedance of cavities with different number of cells obtained by using the ABCI code. The vertical dashed line is at $3 \mathrm{GHz}$. Additional impedance oscillations around fundamental mode are due to wake potential truncation (see Table II). used for impedance calculations of axisymmetric perfectly conducting structures.

\section{A. Cavity impedance}

The impedance of cavities with different number of cells and the fundamental frequency of $400.79 \mathrm{MHz}$ was calculated for two frequency ranges: below $3 \mathrm{GHz}$, which contains "discrete" spectrum, and above $3 \mathrm{GHz}$, the "continuous" spectrum (Fig. 4). We can assume that the cutoff frequencies of all trapped HOMs are below $3 \mathrm{GHz}$ for the beam pipe radius of $150 \mathrm{~mm}$. In superconducting cavities the fundamental mode has a large quality factor $Q \sim 10^{9}$. It means that it is necessary to calculate a very long wake potential to get an accurate impedance value of this mode. In most cases, it is computationally expensive and a truncation of the wake potential should be used. This results in a smaller impedance of the fundamental mode and artificial impedance oscillations around it (see Fig. 4). Thus, the accurate impedance value of the fundamental mode and HOMs below the cutoff frequency can not be obtained, while the resonant frequency and the ratio of the shunt impedance to the quality factor $R / Q$ of these modes can be extracted. The simulation parameters for both frequency ranges are summarized in Table II. To resolve resonances at low frequencies simulations with long bunches and long wakes were used. For higher frequencies it is sufficient to use short wake potentials. It was found that artificial impedance peaks appear at high frequencies for a coarse mesh. Keeping the mesh size of $0.1 \mathrm{~mm}$ allows this problem to be avoided for frequencies below $40 \mathrm{GHz}$.

Comparing the impedances of different cavities, we see that for a larger number of cells per cavity, the total impedance is larger and more passband modes are present. To verify the results obtained with $\mathrm{ABCI}$ code, the loss

TABLE II. Parameters used in ABCI simulations with results shown in Fig. 4.

\begin{tabular}{lcc}
\hline \hline Parameter & $f<3 \mathrm{GHz}$ & $f>3 \mathrm{GHz}$ \\
\hline Bunch length [cm] & 3.6 & 0.1 \\
Wake potential length [m] & 50 & 1 \\
Radial mesh size [mm] & 0.5 & 0.1 \\
Longitudinal mesh size [mm] & 0.5 & 0.1 \\
\hline \hline
\end{tabular}


factor $\kappa_{\|}$was obtained as a function of frequency from the results of CST EM STUDIO® (CST EMS) [8] simulations

$$
\kappa_{\|}(f)=\sum_{n} k_{n} \Theta\left[f-f_{\mathrm{r}, n}\right] e^{-\left(2 \pi f_{\mathrm{r}, n} \sigma\right)^{2}},
$$

where $\Theta$ is the Heaviside step function and the modal loss factor of the $n$th mode is

$$
k_{n}=\pi f_{\mathrm{r}, n}\left(\frac{R}{Q}\right)_{n} .
$$

Here, $f_{\mathrm{r}, n}$ is the frequency of the $n$th resonance mode, $(R / Q)_{n}$ is the ratio of the shunt impedance to the quality factor of this mode. There is a very good agreement between the two codes for frequencies below the cutoff frequency of the $\mathrm{TM}_{010}$ mode $f_{\text {cut }}=2.405 c /(2 \pi d)=$ $765 \mathrm{MHz}$ for the pipe radius $d=15 \mathrm{~cm}$ (Fig. 5). For higher frequencies the difference is due to the fact that the eigenmode solver was used in CST EMS. It requires the structure to be closed at the ends of the beam pipes with either electric or magnetic boundary conditions. This leads to creation of additional resonant modes. Loss factors of these modes significantly depend on the length of the beam pipes indicating their irrelevance for the present study. In practice, such modes can propagate through the beam pipe, have a small loss factor, and contribute to the broadband impedance above cutoff frequency. In ABCI the finitedifference time-domain solver with open (perfectly matched layers) boundaries is implemented. This method prevents the appearing of additional modes as resonances of the closed structure and puts their contribution into the broadband impedance in the correct way. According to simulations for all cavities, only a few resonant modes are present below cutoff frequency. The parameters of these modes are summarized in Table III.

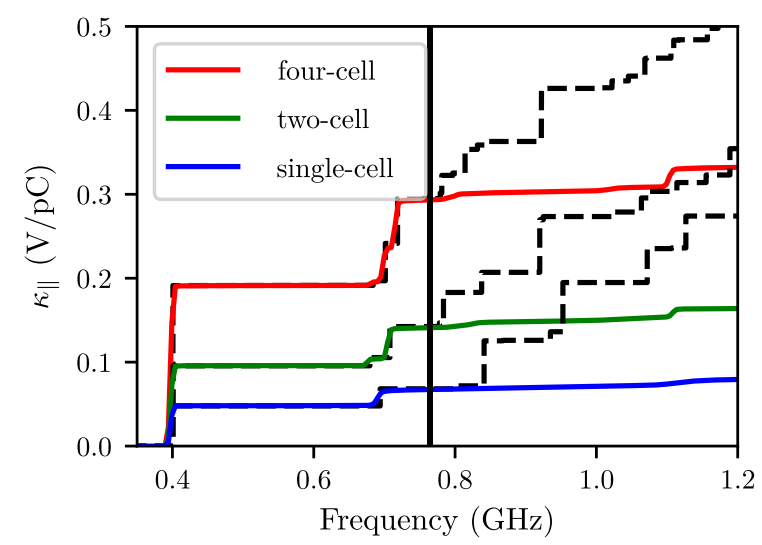

FIG. 5. The loss factor as a function of frequency obtained from ABCI code for different number of cells. The dashed lines are the calculated loss factors from CST EMS results using Eq. (9). The solid vertical line is the cutoff frequency for the beam pipe radius of $15 \mathrm{~cm}\left(f_{\text {cut }}=765 \mathrm{MHz}\right)$.
TABLE III. Parameters of the fundamental mode and HOMs with highest $R / Q$ below the cutoff frequency (CST EMS simulation results). The circuit definition is used for $R / Q$ values.

\begin{tabular}{lcr}
\hline \hline Mode & $f_{\mathrm{r}}[\mathrm{MHz}]$ & $R / Q[\Omega]$ \\
\hline & Single-cell cavity & \\
Fundamental & 400.98 & 42.3 \\
HOM 1 & 694.03 & 11.7 \\
& Two-cell cavity & \\
Fundamental & 400.73 & 84.4 \\
HOM 1 & 679.52 & 4.9 \\
HOM 2 & 707.94 & 22.5 \\
& & \\
Fundamental & Four-cell cavity & 168.5 \\
HOM 1 & 400.72 & 0.2 \\
HOM 2 & 671.27 & 2.6 \\
HOM 3 & 684.06 & 24.7 \\
HOM 4 & 701.38 & 34.8 \\
\hline \hline
\end{tabular}

\section{B. Taper impedance and loss factor}

Tapers are used to make a smooth transition from the large beam pipe radius $d$ inside the cryomodule to the smaller beam pipe radius $b$ outside it. For a fixed ratio $d / b$, the contribution of tapers to the total impedance of the structure depends on taper length $L$. The worst situation is a step transition $(L=0)$, which can result in a significant increase of the longitudinal impedance at frequencies $f \gg c /(2 \pi d)$. The impedance of the step-in and step-out can be calculated analytically in all frequency ranges [9]. For frequencies above cutoff a simple expression can be used [10]

$$
Z_{\text {step }}=\frac{Z_{0}}{\pi} \ln \left(\frac{d}{b}\right)
$$

where $Z_{0}=120 \pi \Omega$ is the impedance of free space.

Simulated impedance spectra (ABCI) of the taper-out for $b=5 \mathrm{~cm}, d=15 \mathrm{~cm}$, and different taper length are shown in Fig. 6. Similarly to the LHC tapers, $b=5 \mathrm{~cm}$ was chosen, because the final decision for the beam pipe radius in the $\mathrm{rf}$ section has not been made. At low frequencies the impedance starts from non-zero value $Z_{\text {step }} / 2$ and then saturates at the value $Z_{\text {step }}$ at higher frequencies. For a stepout $(L=0)$ the spectrum contains resonant peaks at lower frequencies, which disappear for longer tapers. For large $L$, impedance remains constant at low frequencies (for $L=3 \mathrm{~m}$, frequencies are below $10 \mathrm{GHz}$ ) and reaches the saturated value at high frequencies.

The loss factor of a taper-out $\kappa_{\text {taper }}$ as a function of taper length can be approximated as [10],

$$
\kappa_{\text {taper }}=\kappa_{\text {step }} \times\left\{1-\frac{1}{2} \min \left[1, \frac{L}{\tilde{L}}\right]\right\},
$$




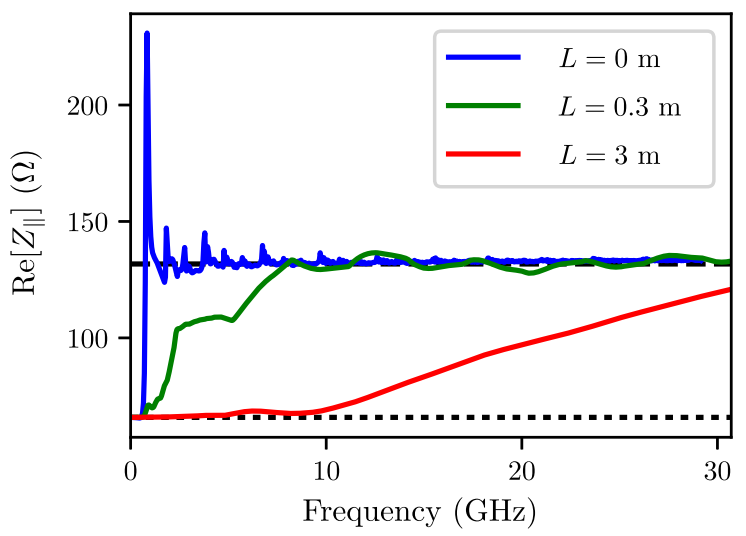

FIG. 6. The impedance spectrum for different lengths of the taper-out (ABCI simulation results). The dashed line is the impedance of the step $Z_{\text {step }}$ [see, Eq. (11)], and the dotted line is $Z_{\text {step }} / 2$.

where the loss factor of a step is

$$
\kappa_{\text {step }}=\frac{Z_{\text {step }}}{2 \sigma \sqrt{\pi}},
$$

and

$$
\tilde{L}=\frac{(d-b)^{2}}{c \sigma}
$$

is the "optimum" length, which corresponds to the minimum value of the taper loss factor. For a bunch length of 12 ps (noncolliding beams in the $\mathrm{Z}$ machine), $\tilde{L} \approx 2.7 \mathrm{~m}$. A smaller value of the loss factor for a longer taper is a result of the lower value of the impedance within the bunch spectrum. Figure 7 shows consistency of the loss factors obtained by $\mathrm{ABCI}$ for different taper lengths with analytic predictions.

To calculate the loss factor of two tapers one has to assume some structure between them. For the simplest

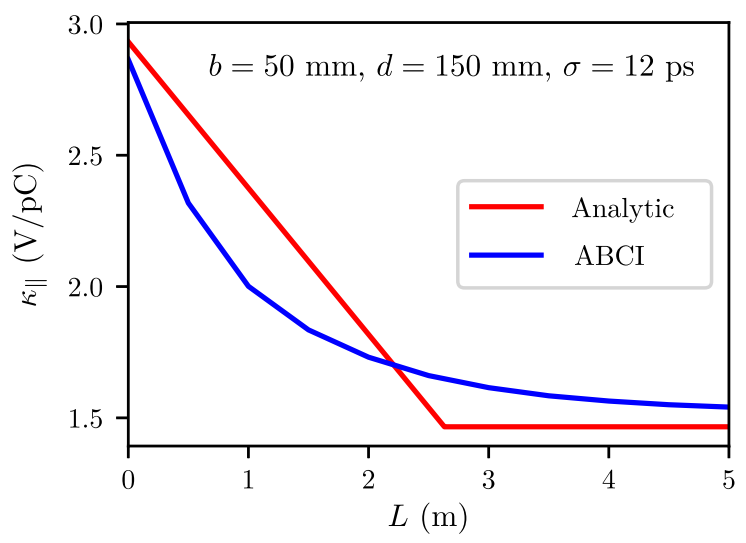

FIG. 7. Comparison of the loss factor of a taper-out from simulations (ABCI) and analytic predictions using Eq. (12) for different taper lengths. case, when a beam pipe of radius $d$ and length $L_{\text {str }}$ is tapered from both sides to radius $b$ using the tapers with length $L$, the analytic expression can be obtained [11]. If $L_{\text {str }}$ fulfills the condition

$$
L_{\mathrm{str}} \gg \frac{d^{2}}{c \sigma}
$$

the loss factor of two tapers is

$$
\kappa_{\text {tapers }}=\frac{Z_{\text {step }}}{2 \sigma \sqrt{\pi}}\left[\frac{2}{\pi} \arctan \left(\frac{0.2 d^{2}}{c \sigma L}\right)\right]^{2} .
$$

For $L \gg d^{2} / c \sigma$ there is a significant reduction of $\kappa_{\text {tapers }}$. This can be understood from the fact that impedances of the taper-in and taper-out are the same but the latter is shifted by $Z_{\text {step }}$ value (Fig. 8). In the sum these impedances the total impedance (the blue line) has a small value for the frequencies containing the bunch spectrum (the black line).

\section{Simulations of full structure}

Considering the case of the full structure which includes cavities and tapers, one needs to clarify if there is a cancellation of the impedance contributions from the taper-in and the taper-out. For results of simulations shown below we assume that four single-cell cavities are separated by a distance of $2 \lambda\left(\lambda=0.748 \mathrm{~m}\right.$ for $\left.f_{\mathrm{rf}}=400.79 \mathrm{MHz}\right)$ and combined in a single cryomodule. This distance can be adjusted in order to have space for HOM couplers and cavity tuners. The tapers $(L=3 \mathrm{~m})$ are placed at a distance $\lambda$ from the center of the last cavities (Fig. 9).

Comparison of the low frequency impedances (below $3 \mathrm{GHz}$ ) of the full structure with step and taper transitions is shown in Fig. 10. There are more resonant lines in the spectrum for the case of step transition than for the case of long tapers. This is due to an additional long cavity

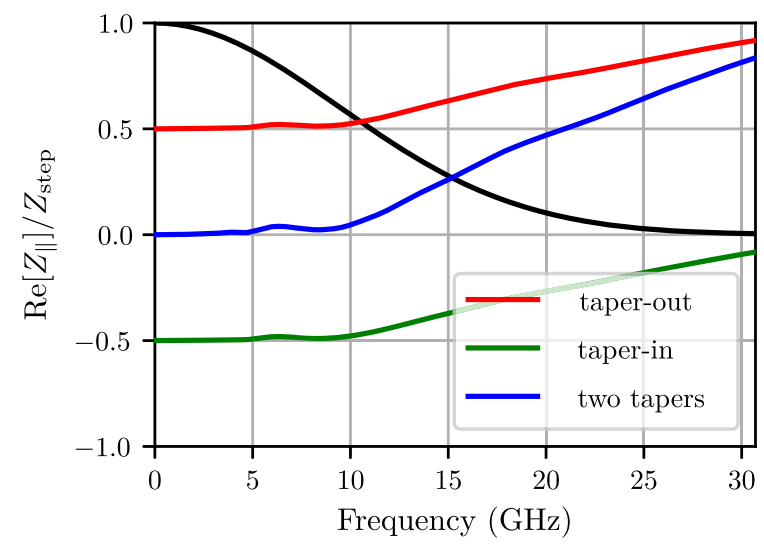

FIG. 8. The normalized impedance spectra of taper-in and taper-out from simulations for $b=50 \mathrm{~mm}, d=150 \mathrm{~mm}$, and $L=3 \mathrm{~m}$. The black line is the envelope of the normalized singlebunch power spectrum for $\sigma=12 \mathrm{ps}$. 


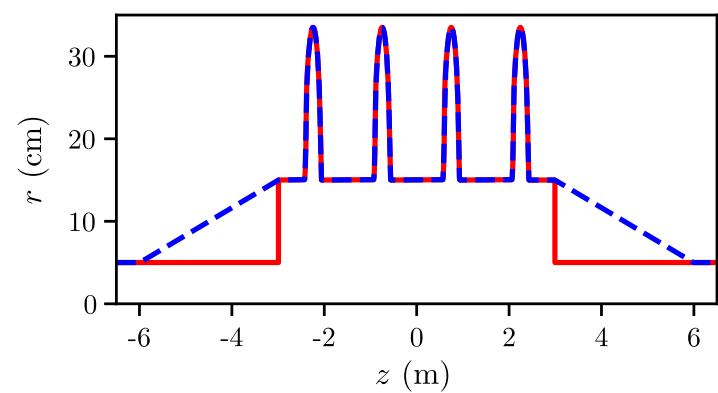

FIG. 9. The full structure of four single-cell cavities with tapers used in simulations.

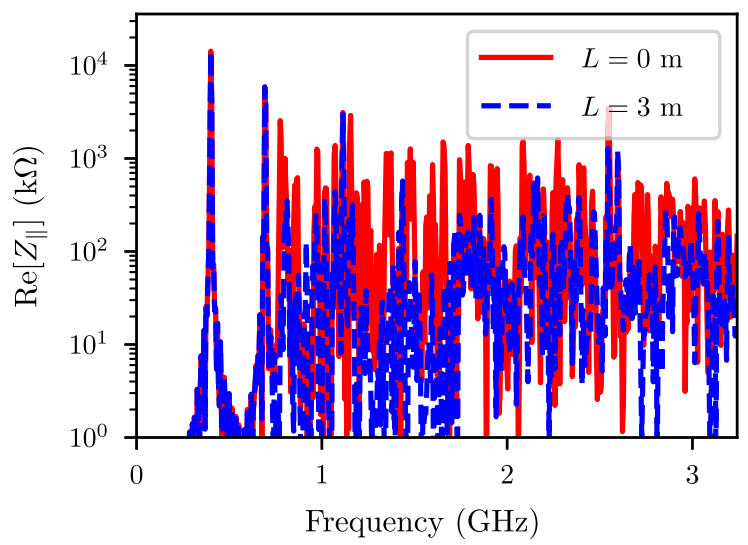

FIG. 10. Impedances of the full structure of four single-cell cavities with step $(L=0)$ and taper transitions (see Fig. 9).

produced by two steps. If we compare the impedance spectra of only four cavities and four cavities with tapers one can see that they are almost identical (Fig. 11). However, there is a difference from the single-cell impedance, which contains fewer resonance lines above the cutoff frequency of $15 \mathrm{~cm}$ beam pipe. This could be due to the

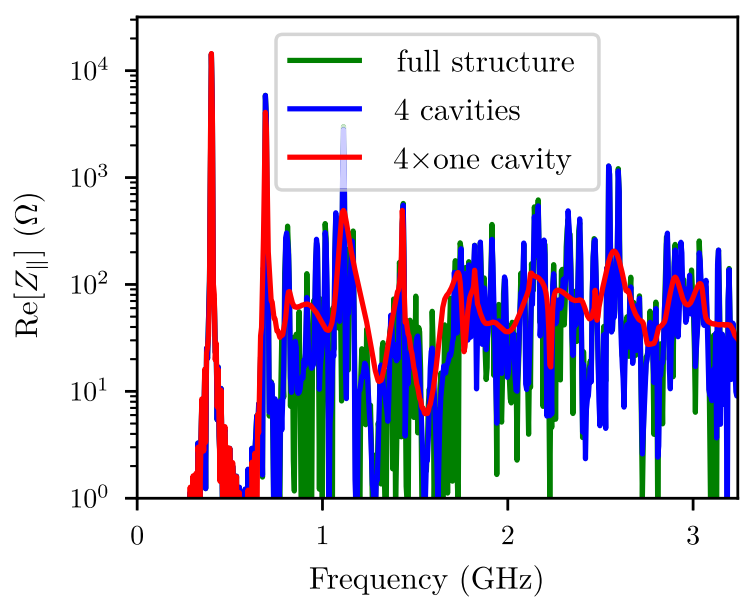

FIG. 11. Comparison of impedance of the full structure containing four single-cell cavities with and without tapers, and of the single-cell cavity multiplied by a factor of 4 .
TABLE IV. The loss factor of different structures obtained with ABCI code. The loss factor of four single-cell cavities is $\kappa_{4 \mathrm{cav}}=1.22 \mathrm{~V} / \mathrm{pC}$. The estimated values are given by Eqs. (13), (12), and (16).

\begin{tabular}{llll}
\hline \hline & \multicolumn{4}{l}{ Loss factor $[\mathrm{V} / \mathrm{pC}]$} & \\
\hline Structure & \multicolumn{4}{c}{ Steps } & Taper-out & Tapers \\
\hline \multicolumn{4}{c}{ Without cavities } \\
Simulations & 3.23 & 1.62 & 0.28 \\
Estimations & 2.93 & 1.47 & 0.17 \\
& \multicolumn{4}{c}{ With cavities } & \\
Simulations & 3.82 & 2.87 & 1.68 \\
Estimations & 4.15 & 2.69 & 1.39 \\
\hline \hline
\end{tabular}

coupling of the HOMs of cavities in the cryomodule, which is not present for a single cavity.

The contributions of different components to the total loss factor of the structure are summarized in Table IV. Without cavities, there are some discrepancies between simulation results and analytic estimations for steps, taper-out, and tapered cavity using Eqs. (13), (12), and (16). This could be due to the resonant modes excited in the long cavity which contribute to the total loss factor. For the case of two tapers the difference is also because a distance between tapers ( $L_{\text {str }} \approx 6 \mathrm{~m}$ ) does not fulfill the condition given by Eq. (15) for a bunch length of $12 \mathrm{ps}\left(d^{2} / c \sigma=5.9 \mathrm{~m}\right)$.

To evaluate the results of the full structure the loss factor of four single-cell cavities was also calculated for the beam pipe without tapering $\left(\kappa_{4 \mathrm{cav}}=1.22 \mathrm{~V} / \mathrm{pC}\right)$. For the step transitions the total loss factor is dominated by the contribution of the steps, but is not given as the sum of $\kappa_{4 \mathrm{cav}}$ and $\kappa_{\text {step }}$. The loss factors of the structures with taper-out and two tapers are significantly smaller in comparison to the case of step transitions. However, contributions of two tapers are not anymore fully compensated as in the case without cavities.

\section{POWER LOSS}

In this section we present the results of the power loss calculations for FCC-ee machines and the cavity impedance obtained with ABCI code. Contributions of the impedance above the cutoff frequency and of the discrete lines are analyzed. For the former case we directly use Eq. (1) with the simulated impedance. For the latter case, only $f_{\mathrm{r}}$ and $R / Q$ can be obtained from simulations, while $Q$ values will be defined by HOM couplers. The power loss calculations and assumptions for the quality factor are discussed in Sec. IV B.

\section{A. Power loss from continuous spectrum}

\section{Dependence on the number of cells}

The power loss calculations for the frequencies above $f_{\text {cut }}$ were done for parameters listed in Table I. The results 


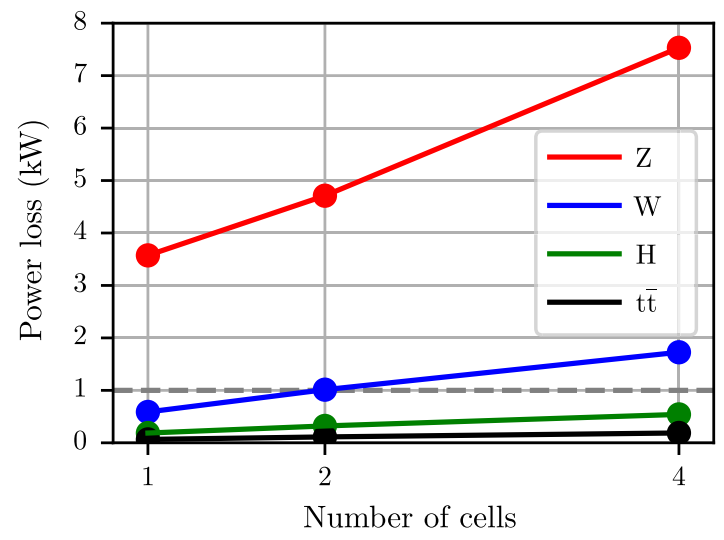

FIG. 12. Power losses for cavities with different number of cells in FCC-ee machines. The horizontal dashed line indicates the maximum power which can be extracted by a single HOM coupler.

for each machine and a single train with the maximum bunch spacing in the ring are summarized in Fig. 12. The $\mathrm{Z}$ machine has the highest power loss due to high beam current. The four-cell cavity design is not practical for the $\mathrm{Z}$ machine because the maximum possible extracted power by a single coupler is around $1 \mathrm{~kW}$ (maximum 6 couplers per cavity). We assume that the HOM couplers are superconducting and terminated with a capacitive vacuum-air feedthrough. This feedthrough is connected inside a vacuum cryostat to a special rf line that brings the HOM power to an rf load at room temperature. The power limit primarily comes from the rf feedthrough and the dimensioning of the vacuum rf lines inside the cryostat to be able to transport $1 \mathrm{~kW}$ safely without inducing a large heat load to the $4.5 \mathrm{~K}$ system. When reaching higher power, other limitations inside the HOM coupler such as multipacting, vacuum breakdown and arcing can also occur. Thus, for the $\mathrm{Z}$ machine the power deposited in a single-cell cavity can be extracted and this option is used for calculations discussed below. For other FCC-ee machines the power losses are below $1 \mathrm{~kW}$ and only for the $\mathrm{W}$ machine parameters in the four-cell cavity it is about $1.5 \mathrm{~kW}$.

Below we analyze the role of different filling schemes for parameters of the $\mathrm{Z}$ machine because of a large total number of bunches. The following parameters are assumed to be constant in calculations: the total beam current, the abort gap length $(2 \mu \mathrm{s})$, the bunch population, the bunch length, and the bunch spacing ( $2.5 \mathrm{~ns})$. Then depending on the train spacing a beam contains different numbers of trains and different numbers of bunches per train. The power loss as a function of $t_{\mathrm{tt}}$ is shown in Fig. 13. The power losses deviate from the value calculated for a single train (the red line) for small distance between trains and then saturate at this value for larger train spacings. The deviations are mostly due to variation of the beam current which changes discretely as a function of the train spacing [see Eq. (8)].

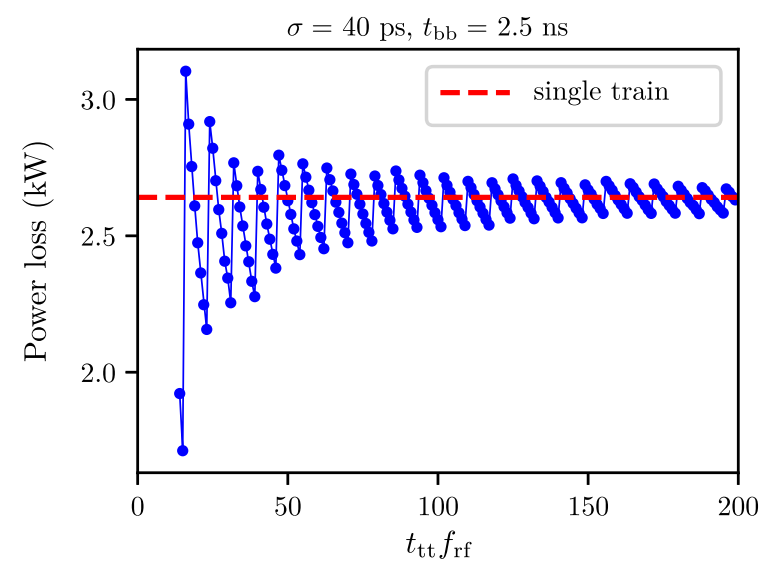

FIG. 13. Dependence of the power loss from continuous impedance spectrum on train spacing. The horizontal dashed line is the value for a single train. Calculations are done for parameters of the $\mathrm{Z}$ machine.

A weak dependence of the power loss on the bunch spacing is visible in Fig. 14. Slightly higher values for larger $t_{\mathrm{bb}}$ are due to overlap of some resonances above cutoff frequency by one of the spectral lines $1 / t_{\mathrm{bb}}$. The power losses for colliding beams $(\sigma=40 \mathrm{ps})$ are still possible to extract by HOM couplers while for noncolliding beams $(\sigma=12 \mathrm{ps})$ this could be difficult.

\section{Contribution of tapers}

The taper loss factor and the optimum length depend on the ratio $d / b$ of larger and smaller pipe radii. For the transition from $d=15 \mathrm{~cm}$ to $b=5 \mathrm{~cm}$ the required taper length is about $3 \mathrm{~m}$ which is not feasible because the taper length would be as long as the length of four cavities in the cryomodule. It means that the taper geometry should be optimized using analytic predictions from Eqs. (12), (14). One of the proposals would be to make transitions to an intermediate beam pipe radius $b_{\text {int }}$ between cryomodules

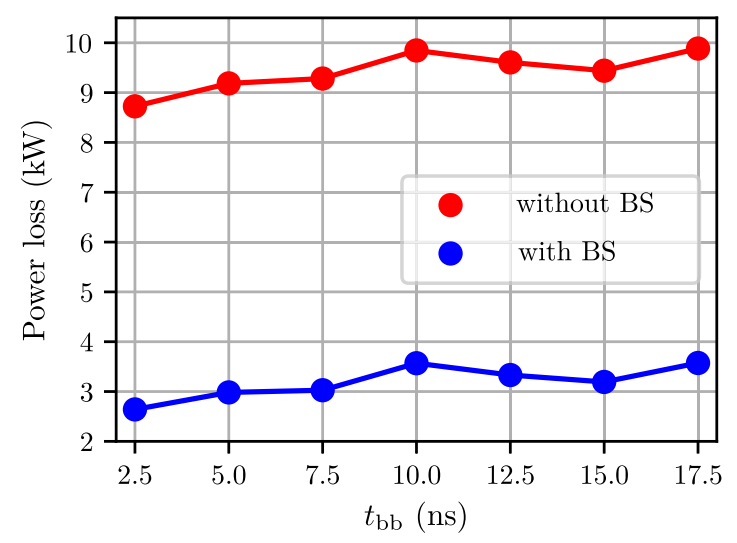

FIG. 14. Power losses for parameters of the $\mathrm{Z}$ machine (colliding and noncolliding beams) as a function of the bunch spacing. 


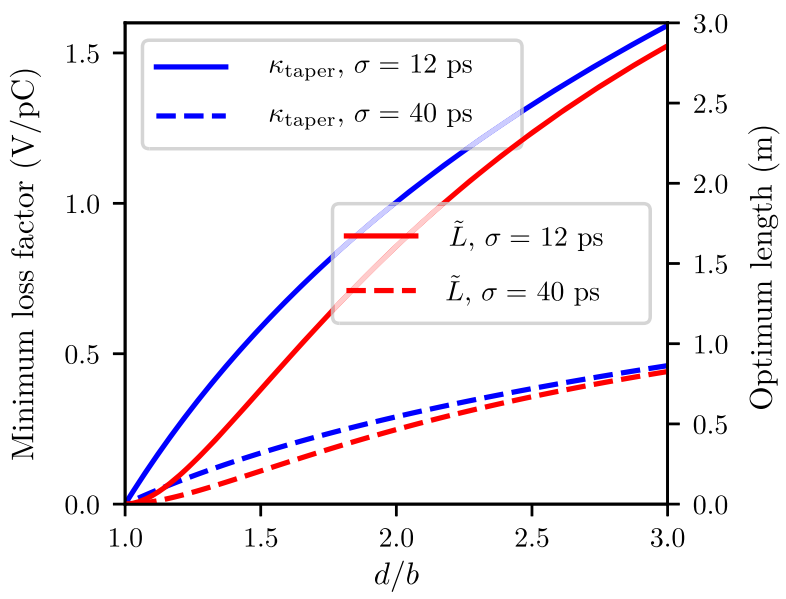

FIG. 15. The minimum loss factor of a taper-out and the optimum taper length as a function of ratio $d / b$ obtained from Eqs. (12), (14) for parameters of noncolliding (solid lines) and colliding (dashed lines) beams in the $\mathrm{Z}$ machine.

(containing 4 cavities) in order to keep the ratio $d / b$ small. For example, for $b_{\text {int }}=10 \mathrm{~cm}$ the required taper length is about $0.8 \mathrm{~m}$, which can fit in the cryomodule (Fig. 15). Then additional tapers from the intermediate radius to beam pipe with radius of $5 \mathrm{~cm}$ can be used only in places where it is necessary (for example, at the end of straight section for the rf system). The intermediate beam pipe radius should be chosen taking into account the aperture size in the quadrupole magnets, vacuum limitations, cold to warm transitions, and the quality factor of the fundamental mode.

According to Eqs. (12), (14), the loss factor and the optimum taper length are inversely proportional to the bunch length. As bunches of colliding beams are almost three times longer than bunches of noncolliding beams, shorter tapers can be used (see Fig. 15).

\section{B. Power loss from discrete cavity spectrum}

\section{Hitting of cavity resonances by bunch spacing beam spectral harmonics}

According to simulations performed with $\mathrm{ABCI}$ and CST EMS, several HOMs are below the cutoff frequency (see Sec. III A). These modes have large $R / Q$ and $Q$ values, and can significantly contribute to the power loss in the case of hitting the high-amplitude beam spectral line.

The worst-case scenario is considered below, when the HOMs sit at the harmonics of the bunch spacing spectral line $1 / t_{\mathrm{bb}}$. We assume first that all HOMs are damped to $Q=10^{4}$ (a typical value for superconducting rf cavities) and evaluate the power loss for all FCC-ee machines using HOM parameters listed in Table III. The results of calculations are summarized in Table V. The highest power losses for all modes are for the $\mathrm{Z}$ machine with $P>100 \mathrm{~kW}$, which means that the cases of hitting the HOM by the bunch spacing spectral lines should be avoided at the cavity design stage. For the $\mathrm{W}$ machine
TABLE V. The worst-case scenario (HOM coincides with the bunch spacing spectral line) for the power loss due to HOMs. Calculations are done for the HOM parameters given in Table III assuming of $Q=10^{4}$.

\begin{tabular}{|c|c|c|c|c|}
\hline \multirow[b]{2}{*}{$\mathrm{HOM}$} & \multicolumn{4}{|c|}{ Power loss [W] } \\
\hline & $\mathrm{Z}$ & W & $\mathrm{H}$ & $\mathrm{t} \bar{t}$ \\
\hline \multicolumn{5}{|c|}{ Single-cell cavity } \\
\hline 1 & $5.0 \times 10^{5}$ & $5.1 \times 10^{3}$ & 290 & 8 \\
\hline \multicolumn{5}{|c|}{ Two-cell cavity } \\
\hline 1 & $2.1 \times 10^{5}$ & $2.1 \times 10^{3}$ & 120 & 3 \\
\hline 2 & $9.6 \times 10^{5}$ & $9.8 \times 10^{3}$ & 560 & 16 \\
\hline \multicolumn{5}{|c|}{ Four-cell cavity } \\
\hline 1 & $7.4 \times 10^{3}$ & 75 & 4 & 0.1 \\
\hline 2 & $1.1 \times 10^{5}$ & $1.1 \times 10^{3}$ & 64 & 2 \\
\hline 3 & $1.1 \times 10^{6}$ & $1.1 \times 10^{4}$ & 610 & 17 \\
\hline 4 & $1.5 \times 10^{6}$ & $1.5 \times 10^{4}$ & 870 & 24 \\
\hline
\end{tabular}

the power loss $P$ can be up to $15 \mathrm{~kW}$ for $Q=10^{4}$, while for $Q=10^{3}$ the maximum power loss can be kept below $1.5 \mathrm{~kW}$. As the high-energy machines $(\mathrm{H}$ and $t \bar{t})$ have smaller beam current, the power loss is significantly lower and can be easily extracted by HOM couplers.

In cases of high shunt impedance, to maintain longitudinal beam stability reduction of the HOM quality factor is necessary. So that the corresponding instability growth time $\tau_{\text {inst }}$ becomes longer than the synchrotron radiation damping time $\tau_{\mathrm{SR}}$. For the case of longitudinal coupled bunch instability, one can use the following estimation [12]

$$
\tau_{\mathrm{inst}}=\frac{2 E Q_{s}}{e|\eta| J_{A} f_{\mathrm{r}}(R / Q) Q}>\tau_{\mathrm{SR}}
$$

where $\eta=\alpha_{p}-1 / \gamma^{2}$ is the slip factor. Equation (17) gives the maximum value of the shunt impedance at a given resonant frequency $f_{\mathrm{r}}$ when the beam is stable. As the growth time is inversely proportional to the beam current it is the shortest for the $\mathrm{Z}$ machine with the longest $\tau_{\mathrm{SR}}$. For this machine we consider a single-cell cavity design with the accelerator gradient around $2 \mathrm{MV} /$ cavity. To get the total accelerating voltage of $100 \mathrm{MV}$ the total number of cavities should be 52 because they are combined by four in cryomodules. This gives the limit for the shunt impedance as a function of frequency $5.2 \mathrm{k} \Omega / f_{\mathrm{r}}[\mathrm{GHz}]$, and the HOM of the single-cell cavity should be damped to $Q=640$. In this case, the power loss is about $30 \mathrm{~kW}$ if the bunch spacing spectral line hits the HOM and difficult to handle.

\section{Power loss for different filling schemes}

As was already discussed in Sec. II, in the presence of trains the beam spectrum also contains $1 / t_{\mathrm{tt}}$ spectral lines. Some of these lines can hit the cavity resonances and lead to significant power losses. Here we discuss how to identify 


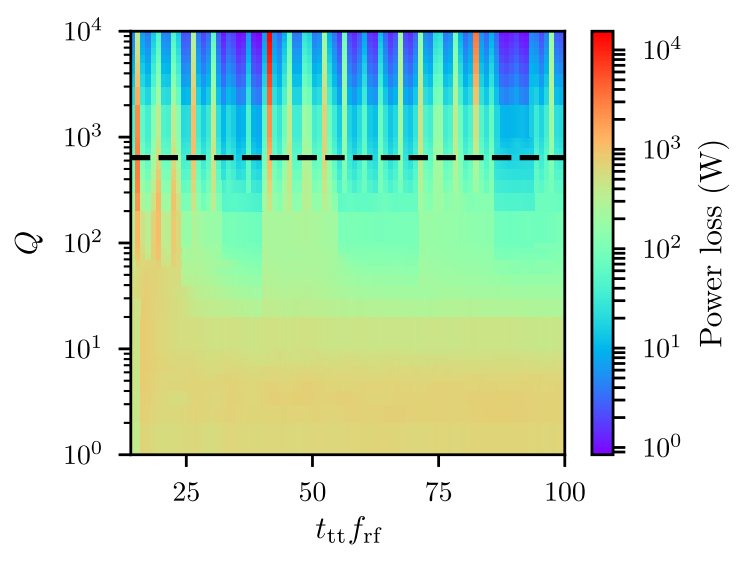

FIG. 16. Power losses from the HOM in the single-cell cavity (mode 1 in Table III) for different train spacings $t_{\mathrm{tt}}$ and different quality factors $Q$. Calculations are done for parameters of the $\mathrm{Z}$ machine with $2.5 \mathrm{~ns}$ bunch spacing. The dashed black line indicates the quality factor required for the longitudinal beam stability.

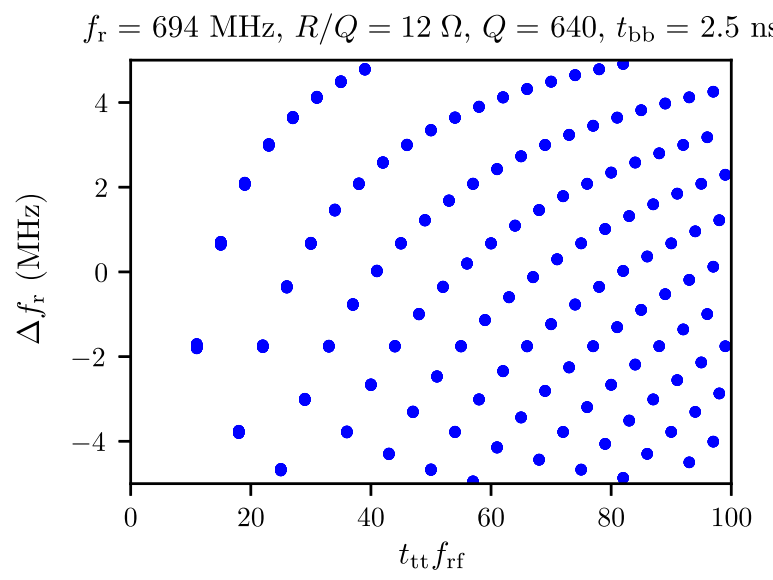

FIG. 17. Cases when the spectral line hits the HOM with frequency variation $\pm 5 \mathrm{MHz}$. Calculations are done for the $\mathrm{Z}$ machine parameters using Eq. (18). such situations, and finally define recommendations for the cavity design and machine filling schemes.

We focus on the power loss in the $\mathrm{Z}$ machine for the single-cell cavity design as it has the highest power losses from continuous spectrum and in the case when HOM is hit by one of $1 / t_{\mathrm{bb}}$ spectral lines. The power loss map calculated by varying the distance between trains $t_{\mathrm{tt}}$ and the quality factor of the HOM (mode 1 in Table III) is shown in Fig. 16. For small $Q$, power losses are around $1 \mathrm{~kW}$ and do not depend on $t_{\mathrm{tt}}$. In this case the impedance is broadband and overlaps with many beam spectral lines. Thus the HOM should not be damped below certain level necessary for beam stability. For medium $Q$, moderate losses around a few hundreds of watts are observed. For high $Q$, there are several values of $t_{\mathrm{tt}}$ with large power losses. This corresponds to the situation when one of the spectral lines hits the resonance impedance.

To identify the cases when the additional spectral lines hit the resonant line (below referred to as "resonant" cases), we propose to use the following equation

$$
\left|1-\frac{\left[f_{\mathrm{r}} t_{\mathrm{tt}}\right]}{f_{\mathrm{r}} t_{\mathrm{tt}}}\right|<\frac{1}{Q}
$$

where $\left[f_{\mathrm{r}} t_{\mathrm{tt}}\right]$ denotes the rounded off value. Due to manufacturing uncertainties each cavity will have slightly different frequencies of HOMs, this fact is taken into account in the power loss calculation presented below. For each train spacing we vary the frequency of the considered mode with a maximum shift $\Delta f_{\mathrm{r}}$ and identify resonant cases using Eq. (18). We see that many resonant cases are present (Fig. 17), but one should not expect a high power loss for all train spacings. To demonstrate this we make a scan of the resonant frequency $f_{\mathrm{r}}=694 \pm 5 \mathrm{MHz}$ with a step of $1 \mathrm{kHz}$ of the damped mode $(R / Q=12 \Omega$ and $Q=640$ ) for different filling schemes. For each train spacing the highest power loss is shown the in Fig. 18.

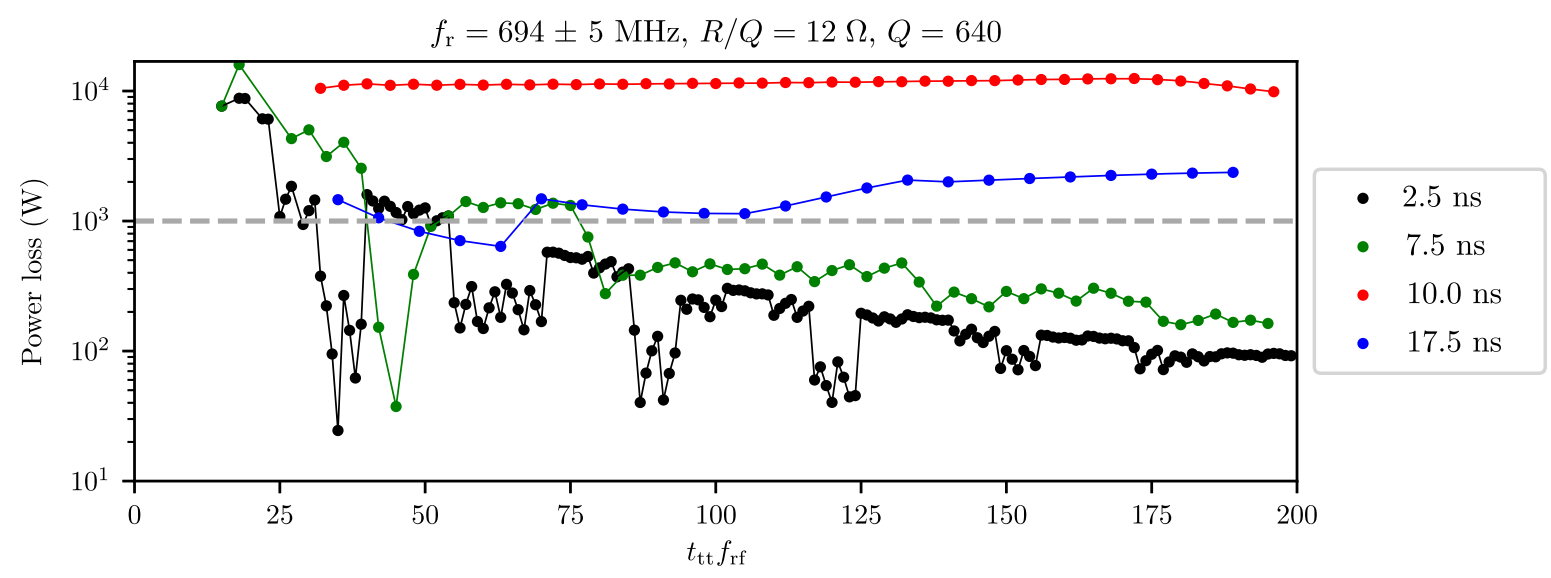

FIG. 18. Dependence of the maximum power loss in resonant cases on the train spacing for the parameters of the $\mathrm{Z}$ machine and different bunch spacings. The horizontal line shows the limit of $1 \mathrm{~kW}$. For bunch spacings $t_{\mathrm{bb}}=5,12.5,15$ ns the behavior of the power losses is similar to $t_{\mathrm{bb}}=2.5 \mathrm{~ns}$ (the black dots) and the power losses are below the values corresponding to $t_{\mathrm{bb}}=7.5 \mathrm{~ns}$. 
The power losses are always above $1 \mathrm{~kW}$ for $t_{\mathrm{bb}}=10 \mathrm{~ns}$ and $t_{\mathrm{bb}}=17.5 \mathrm{~ns}$ since one of the $1 / t_{\mathrm{bb}}$ spectral lines is close to the HOM. It means that for the present single-cell cavity design these bunch spacings are not acceptable. For other bunch spacings power losses are above $1 \mathrm{~kW}$ for $t_{\mathrm{tt}} f_{\mathrm{rf}}<75$ which means that these filling schemes should be avoided in machine operation. For larger train spacings power losses are below $1 \mathrm{~kW}$ because the amplitude of additional spectral lines decreases for smaller number of trains $n_{\text {tr }}$, see Eq. (5).

\section{Recommendations for new cavity designs}

If the HOM frequency is far enough from one of the $1 / t_{\mathrm{bb}}$ spectral lines, significant power losses can be avoided. This defines a "safe" frequency range with a small power loss for a given bunch spacing. To identify this region we place the resonance frequency between two bunch spacing spectral lines, vary the frequency shift $\Delta f_{\mathrm{r}}$, and calculate the power loss as a function of the train spacing. For larger frequency shifts the larger train spacings are required to keep power losses below $1 \mathrm{~kW}$ according to calculations with $t_{\mathrm{bb}}=2.5 \mathrm{~ns}$ (Fig. 19). It means that if the power loss limit is fixed, the number of train spacings which should be avoided in operation depends on $\Delta f_{\mathrm{r}}$.

Thus, a certain criteria to fix either $\Delta f_{\mathrm{r}}$ or $t_{\mathrm{tt}}$ should be used. According to Eq. (8) the total beam current depends on the train spacing which is demonstrated in Fig. 20. The $5 \%$ deviation of the beam current from the nominal value leads to the condition $t_{\mathrm{tt}} f_{\mathrm{rf}}>100$. Assuming that short train spacings $\left(t_{\mathrm{tt}} f_{\mathrm{rf}}<100\right)$ should be avoided in operation, frequency ranges with acceptable power losses for all bunch spacings can be defined. The results are shown in

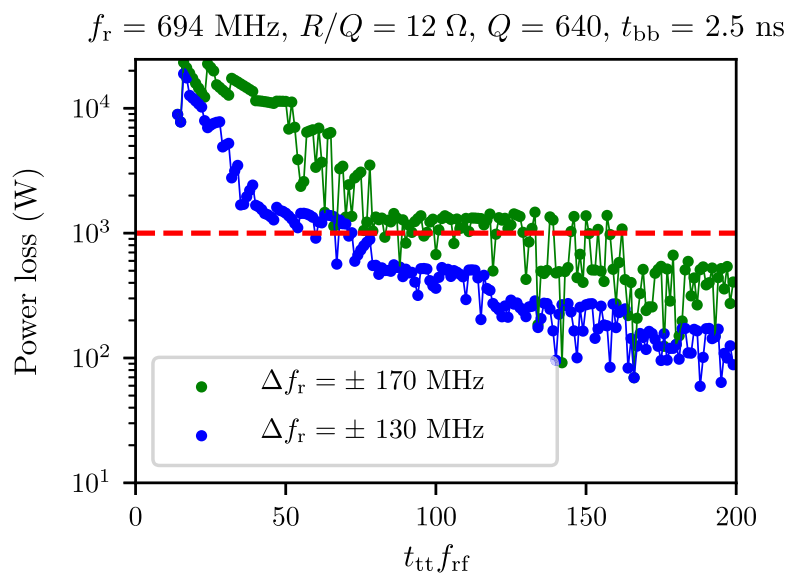

FIG. 19. Dependence of the maximum power loss in the resonant cases on the train spacing for different frequency shifts of HOM. For each train spacing the frequency of the mode was varied with the step of $3 \mathrm{kHz}$. For each train spacing, results are obtained from the scan of the resonant frequency with a step of $10 \mathrm{kHz}$. The horizontal line corresponds to the HOM coupler limit of $1 \mathrm{~kW}$.

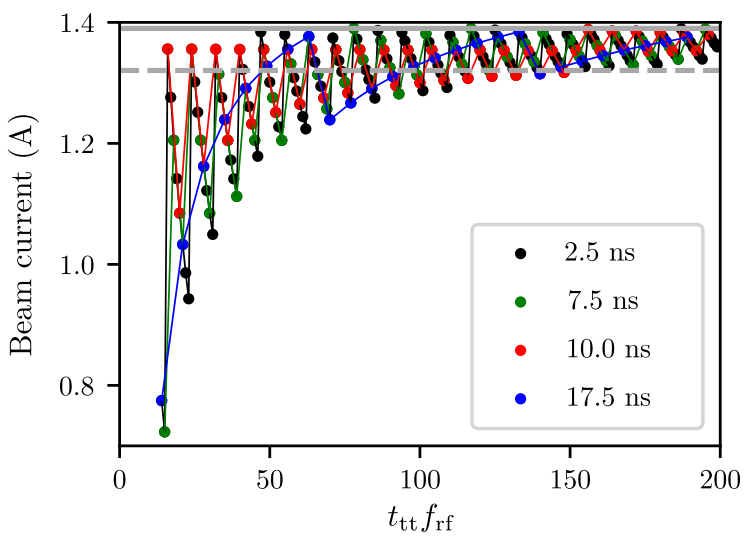

FIG. 20. Dependence of the total beam current in the resonant cases on the train spacing for different bunch spacings. The solid line corresponds to the design current $J_{A}=1.39 \mathrm{~A}$, and the dashed line is at $0.95 \times J_{A}$.

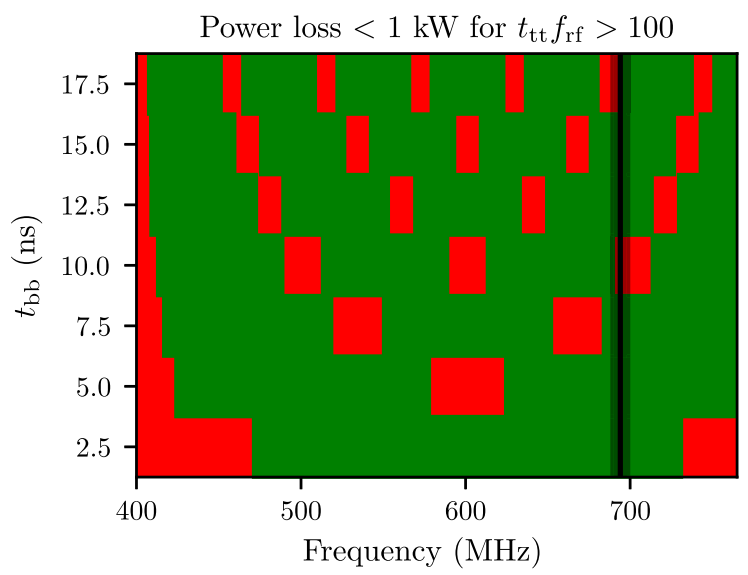

FIG. 21. Frequency ranges with acceptable power loss below $1 \mathrm{~kW}$ for different bunch spacings (green regions). Regions with power loss above $1 \mathrm{~kW}$ are shown with red color. The vertical black line corresponds to the position of HOM in the single-cell cavity with the maximum frequency shift of $\pm 5 \mathrm{MHz}$. The considered frequency range is limited by the frequency of the fundamental mode $(400.79 \mathrm{MHz})$ and the cutoff frequency (765 MHz).

Fig. 21. These regions are different for different $t_{\mathrm{bb}}$. One can see also that it is difficult to find the frequency of HOM which is suitable for all bunch spacing (see, for example, the position of HOM for the present single-cell cavity design). By excluding one of the possible bunch spacings these regions can be expanded giving more flexibility for possible cavity designs.

\section{CONCLUSIONS}

Accurate knowledge of high beam-induced power losses for accelerating structures is necessary to avoid damage of their components. In this work semianalytical and simulation tools were used to evaluate the cavity designs for FCC-ee machines with this objective. All considered 
options (single-cell, two-cell, and four-cell cavities) could be used in $\mathrm{W}, \mathrm{H}, \mathrm{t} \overline{\mathrm{t}}$ machines because power losses from cavity impedance above cutoff frequency are below or around $1.5 \mathrm{~kW}$.

The $\mathrm{Z}$ machine is the most challenging due to high total beam current and large number of bunches. Thus, a singlecell cavity is the only feasible option. The power losses above cutoff frequency weakly depend on the bunch spacing due to overlap of some resonances with one of the bunch spacing spectral lines. The contribution of tapers has to be minimized by using tapers with proper dimensions.

We have identified the cases when high-amplitude beam spectral lines defined by the train spacing can overlap with the HOM below cutoff frequency. The results of a systematic scan show that $10 \mathrm{~ns}$ and $17.5 \mathrm{~ns}$ bunch spacings are not feasible due to strong power losses. For other bunch spacings, the train spacings smaller than 75 rf buckets should be avoided in operation.

Finally, for the train spacings longer than 100 rf buckets the frequency range for the considered HOM of the singlecell cavity was found where power losses are below $1 \mathrm{~kW}$. The width of these frequency ranges depends on the bunch spacing. The overlap of safe regions is possible by excluding some of the bunch spacings in operation. This strategy can be used as recommendation for new cavity designs with a range for acceptable HOM frequencies defined by requirements from machine operation.

\section{ACKNOWLEDGMENTS}

We would like to thank Andrew Butterworth, Olivier Brunner and Nikolay Schwerg for useful discussions and collaboration. The special acknowledgments go to Juan Esteban Müller who previously worked on this topic and to Simon Albright for reading this paper. We also thank Dmitry Teytelman and Robert Rimmer for the useful comment about filling schemes.
[1] A. W. Chao, Physics of Collective Beam Instabilities in High Energy Accelerators (John Wiley \& Sons, Inc., New York, 1993), p. 236.

[2] B. W. Zotter and S. Kheifets, Loss factors and effective impedances, in Impedances and Wakes in High Energy Particle Accelerators (World Scientific, Singapore, 1998), p. 97.

[3] K. Oide (on behalf of FCC-ee Collaboration), FCC-ee Optics Update, The fourth Annual Meeting of the Future Circular Collider Study, Amsterdam (2018), https://indico .cern.ch/event/656491/contributions/2939185/attachments/ 1628880/2597276/FCCee_Optics_Oid e_180411.pdf.

[4] F. Caspers, C. Gonzalez, M. D’yachkov, E. Shaposhnikova, and H. Tsutsui, CERN Report No. PS/RF/Note 2000-004, 2000, http://cdsweb.cern.ch/record/960191/files/cer-00262 6476.pdf.

[5] R. Calaga, A. Butterworth, O. Brunner, and E. Jensen, SRF for future circular colliders, in Proceedings of 17th International Conference on $R F$ Superconductivity (Whistler, BC, Canada, 2015), http://srf2015proc.triumf .ca/prepress/papers/frba04.pdf.

[6] ABCI (Azimuthal Beam Cavity Interaction) code, http:// abci.kek.jp/abci.htm.

[7] D. Boussard and T. Linnecar, The LHC superconducting RF system, in Proceedings of Cryogenic Engineering and International Cryogenic Materials Conference (Montreal, Canada, 1999), https://cds.cern.ch/record/410377.

[8] CST EM STUDIO, https://www.cst.com/products/cstems.

[9] A. M. Al-Khateeb, Longitudinal geometric loss factor and impedance of a step-out discontinuity at arbitrary beam energy in a round cylindrical beam-pipe, Nucl. Instrum. Methods Phys. Res., Sect. A 635, 35 (2011).

[10] S. A. Heifets and S. A. Kheifets, Coupling impedance in modern accelerators, Rev. Mod. Phys. 63, 631 (1991).

[11] A. Blednykh and S. Krinsky, Loss factor for short bunches in azimuthally symmetric tapered structures, Phys. Rev. ST Accel. Beams 13, 064401 (2010).

[12] K. Y. Ng, Longitudinal coupled-bunch instabilities, in Physics of Intensity Dependent Beam Instabilities (World Scientific, Singapore, 2006), p. 311. 